\title{
Respuestas psicofisiológicas ante estímulos asociados al tabaco: diferencias entre fumadores y no fumadores
}

\author{
Psychophysiological Responses to Tobacco Related Stimuli: \\ Differences between Smokers and non-Smokers \\ Respostas psicofisiológicas ante estímulos associados ao tabaco: \\ diferenças entre fumadores e não fumadores
}

\author{
Carlos Gantiva*, Yeraldín Ballén*, María Casas*, Katherine Camacho* \\ *Universidad de San Buenaventura, Bogotá, Colombia.
}

Doi: http://dx.doi.org/10.12804/apl34.3.2016.09

\section{Resumen}

Las respuestas emocionales y motivacionales son fundamentales para explicar el inicio y mantenimiento del consumo de tabaco; sin embargo, las diferencias en fumadores y no fumadores de las respuestas psicofisiológicas que sirven como marcadores neurofisiológicos de procesos emocionales y motivacionales presentan resultados contradictorios. El objetivo de esta investigación fue identificar las diferencias en las respuestas psicofisiológicas ante estímulos asociados al tabaco en fumadores y no fumadores. Para esto se registró el reflejo de sobresalto y la respuesta galvánica de la piel mientras observaban imágenes apetitivas, neutrales, aversivas y asociadas al tabaco. Los resultados muestran que los fumadores inhiben el reflejo de sobresalto ante las imágenes asociadas al tabaco de forma similar que ante las imágenes apetitivas; así mismo, se encontró en los fumadores una mayor inhibición del reflejo de sobresalto ante las imágenes de tabaco. No se encontraron diferencias en la respuesta galvánica de la piel. Estos resultados sugieren que los fumadores activan el sistema motivacional apetitivo ante los estímulos asociados al tabaco.

Palabras clave: tabaco; emoción; motivación; reflejo de sobresalto; fumadores.

\section{fbstract}

Emotional and motivational mechanisms have fundamental to explain the initiation and maintenance of tobacco use, however, results respect to differences in psychophysiological responses which serve as neurophysiological markers of emotional and motivational processes in smokers and nonsmokers have mixed results. The aim of the present study was to identify differences in psychophysiological responses to tobacco related stimuli in smokers and non-smokers, for this

* Carlos Gantiva, Yeraldín Ballén, María Casas, Katherine Camacho, Programa de Psicología, Universidad de San Buenaventura, Bogotá, Colombia.

Este estudio fue financiado por la Universidad de San Buenaventura, Bogotá (Ref. PSI 010-004).

La correspondencia relacionada con este artículo debe ser dirigida a Carlos Gantiva, Programa de Piscología, Universidad de San Buenaventura, Bogotá, Colombia, Cr 8H \# 172-20. Correos electrónicos: cgantiva@usbbog.edu.co, cgantiva@gmail.com

Cómo citar este artículo: Gantiva, C., Ballén, Y., Casas, M., \& Camacho, K. (2016). Respuestas psicofisiológicas ante estímulos asociados al tabaco: diferencias entre fumadores y no fumadores. Avances en Psicología Latinoamericana, 34(3), $557-564$. doi: http://dx.doi.org/10.12804/ap134.3.2016.09 
startle reflex and galvanic skin response was recorded while they were viewing pleasant, neutral, unpleasant and tobacco-related pictures. The results show that smokers inhibit the startle reflex to tobacco-related pictures in a similar way to pleasant pictures, additionally; smokers have greater inhibition of the startle reflex to tobacco-related pictures. No significant differences were found between groups in galvanic skin response. It is concluded that smokers activate the appetitive motivational system to cigarette-related cues.

Keywords: tobacco; emotion; motivation; startle reflex; smokers.

\section{Resumo}

As respostas emocionais e motivacionais são fundamentais para explicar o início e manutenção do consumo de tabaco, no entanto, os resultados com respeito às diferenças em fumadores e não fumadores das respostas psicofisiológicas que servem como marcadores neurofisiológicos de processos emocionais e motivacionais apresentam resultados contraditórios. O objetivo desta pesquisa foi identificar as diferenças nas respostas psicofisiológicas ante estímulos associados ao tabaco em fumadores e não fumadores, para isto se registrou o reflexo de sobressalto e a resposta galvânica da pele enquanto observavam imagens apetitivas, neutrais, aversivas e associadas ao tabaco. Estes resultados sugerem que os fumadores ativam o sistema motivacional apetitivo ante os estímulos associados ao tabaco.

Palavras-chave: tabaco; emoção; motivação; reflexo de sobressalto; fumadores.

El consumo de tabaco ha sido considerado por la Organización Mundial de la Salud (OMS) como uno de los principales problemas de salud pública. Se calcula que alrededor de 6 millones de personas mueren al año por causas asociadas al consumo de tabaco (OMS, 2011). Según los datos oficiales de la OMS, la prevalencia de consumo en mayores de 15 años en países latinoamericanos como Argentina es del $22 \%$, en Brasil de 13\%, en Chile de 33\%, en
México de 8\%, en Estados Unidos la prevalencia es del $25 \%$ y en países europeos como España y Francia es del 27\% (OMS, 2014).

La respuesta hacia los estímulos asociados al tabaco es una de las variables fundamentales para comprender las causas del consumo y las recaídas durante el proceso de abandono (Shiffman et al., 2007). Se ha identificado que los fumadores con baja motivación al cambio evalúan como más apetitivas y activantes las imágenes asociadas al tabaco que los fumadores con alta disposición al cambio (Gantiva, Rodríguez, Arias, \& Rubio, 2012a; Muñoz et al., 2010), y que los fumadores presentan mayor amplitud de potenciales positivos tardíos (Versace et al., 2011; Versace et al., 2010) y sesgos atencionales (Bradley, Mogg, Wright, \& Field, 2003) ante estímulos asociados al tabaco. Sin embargo, los resultados con respecto a los procesos emocionales y motivacionales utilizando medidas objetivas, como el reflejo de sobresalto y la respuesta galvánica de la piel, han sido contradictorios.

El estudio objetivo de la motivación es posible a partir de la modulación del reflejo de sobresalto, el cual es un reflejo defensivo que se puede generar a partir de un ruido aversivo e inesperado y se mide a partir de la electromiografía (EMG) del músculo orbicular (Blumenthal et al., 2005). Se ha comprobado que cuando se observan imágenes aversivas el reflejo de sobresalto aumenta y disminuye ante la visualización de imágenes apetitivas (Bradley, Codispoti, Cuthbert, \& Lang, 2001; Bradley \& Lang, 2007; Gantiva, Díaz, Ospina, Gutierrez, \& Romo-González, 2014). Este fenómeno es denominado "modulación del reflejo de sobresalto" y suele ser estudiado a partir de la metodología de visualización de imágenes afectivas (Gantiva, Guerra, \& Vila, 2015; Lang, 1995, 2010).

Las investigaciones que han estudiado la modulación del reflejo de sobresalto ante estímulos asociados al tabaco han mostrado diferentes resultados. Por ejemplo, Cinciripini et al. (2006) y Dempsey, Cohen, Hobson y Randall (2007) encontraron que los fumadores inhiben el reflejo de 
sobresalto ante imágenes asociadas al tabaco; esto sugiere la activación del sistema motivacional apetitivo, lo que a su vez se relaciona con conductas de aproximación, sin embargo, también se han encontrado resultados contradictorios utilizando esta misma metodología y población (Muñoz et al., 2010). Otros estudios han mostrado diferencias en el reflejo de sobresalto según el grado de dependencia a la nicotina y el nivel de consumo de tabaco (Cui et al., 2012; Rehme et al., 2009), pero entre estos estudios también hay contradicciones.

Otra variable fundamental para comprender la respuesta emocional es el arousal, lo cual se refiere a la intensidad de la emoción experimentada y está en función de la valencia del estímulo. Se ha demostrado con medidas subjetivas y objetivas que el arousal aumenta ante estímulos apetitivos y aversivos, y disminuye ante estímulos neutrales (Bradley, 2009; Bradley et al., 2001; Gantiva, Guerra, \& Vila, 2011). Objetivamente el arousal es medido a través de la respuesta galvánica de la piel (GSR); se ha observado que los fumadores responden con mayor GSR ante videos asociados al consumo de tabaco que ante videos con contenido neutral (Tong, Bovbjerg, \& Erblich, 2007).

Debido a los resultados ambiguos con respecto a la modulación del reflejo de sobresalto y a la poca evidencia de los cambios de la GSR ante imágenes asociadas al tabaco en fumadores, así como a la ausencia de este tipo de estudios en población latinoamericana, el objetivo de esta investigación fue identificar la magnitud de la modulación del reflejo de sobresalto y la GSR ante imágenes asociadas al tabaco en fumadores y no fumadores, lo que permitirá identificar objetivamente las diferencias en los procesos motivacionales y emocionales entre estas dos poblaciones. Los resultados aumentarán la comprensión del comportamiento de consumo, permitirán desarrollar procesos de intervención y evaluar, a través de esta metodología, la efectividad de tratamientos para el abandono del consumo de tabaco, como se ha logrado en el consumo de alcohol. Por ejemplo, Loeber et al.
(2007) encontraron en pacientes alcohólicos que la modulación del reflejo de sobresalto ante imágenes asociadas al alcohol era un mejor predictor de recaídas en comparación con medidas de autoreporte y de activación autónoma. Recientemente, Marín, Jurado, Martínez, Ponce y Rubio (2014) encontraron que la modulación del reflejo de sobresalto puede ser considerada una variable neurofisiológica que constituye un marcador de vulnerabilidad para el desarrollo del alcoholismo o el resultado de los efectos neurotóxicos que el alcohol ejerce sobre el sistema nervioso central.

\section{Método}

\section{Participantes}

El estudio se desarrolló con 60 personas voluntarias, 26 hombres (21-29 años $M$ edad: 24,32, $D E$ : $3,14)$ y 34 mujeres $(22-28$ años; $M$ edad: 23,62 , $D E: 2,86)$, distribuidas en dos grupos de 30 personas cada uno de fumadores y no fumadores. Todos los participantes tienen un nivel educativo mínimo de secundaria y no reportan historia de trauma craneoencefálico o trastorno neurológico. Ningún participante estaba bajo tratamiento psicológico ni farmacológico y se excluyeron personas con déficit no corregidos a nivel auditivo o visual. Los participantes del grupo de fumadores tienen una historia de consumo de tabaco de al menos un año $(M=4,31$ años, $D E=1,03$ años) y un consumo promedio de 7,4 cigarrillos al día $(D E=1,22)$; el grupo de no fumadores reportan no haber fumado nunca en su vida.

\section{Estímulos y procedimiento}

Se utilizaron un total de 28 imágenes a color. De éstas, 21 fueron seleccionadas del Sistema Internacional de Imágenes Afectivas ${ }^{1}$ (IAPS) (Lang,

1 Los códigos de las imágenes del IAPS fueron: apetitivas 4676, 4693, 4668, 4692, 4698, 4623, 4677; neutrales 7026, $7161,7179,7013,7018,7041,7021$; aversivas 1525,1932 , $6315,6520,9163,3213,2683$. 
Bradley, \& Cuthbert, 2008) a partir de los valores normativos de la población colombiana (Gantiva et al., 2011), y 7 imágenes relacionadas al tabaco (celebraciones sociales, tiempo de ocio, cigarrillos y paquetes) con alta valencia apetitiva y alto arousal en población colombiana (Gantiva et al., 2012b). Cada imagen fue presentada durante 6 segundos en una pantalla plana de 19 pulgadas localizada a $60 \mathrm{~cm}$ del participante. Todas las imágenes fueron presentadas dos veces en tres órdenes contrabalanceados de 56 imágenes cada uno.

En cada bloque las imágenes fueron presentadas en orden aleatorio y el intervalo entre ensayos variaba aleatoriamente entre 10 a 14 segundos. El estímulo sonoro utilizado para generar el reflejo de sobresalto fue un ruido blanco de 50 milisegundos de duración, de $105 \mathrm{~dB}$ y con tiempo de subida instantáneo, administrado entre los segundos $4 \mathrm{y}$ 5,5 después del inicio de la imagen (Blumenthal et al., 2005). El sonido de sobresalto se presentó aleatoriamente en la mitad de los ensayos de cada categoría de imagen y en cuatro intervalos entre ensayos.

\section{Equipos y medición de las respuestas psicofisiológicas}

Para presentar los estímulos visuales y auditivos se utilizó el software E-Prime, versión 2.0 (Psychology Software Tools, Pennsylvania, USA). Para registrar la actividad electromiográfica y la GSR se utilizó un equipo PowerLab 26T (ADInstruments) y el software LabChart (v7.3, ADInstruments, Australia). El reflejo de sobresalto se midió a través de dos electrodos de $4 \mathrm{~mm} \mathrm{Ag/AgCl}$ colocados sobre el músculo orbicular del ojo izquierdo con una tasa de muestreo de $1000 \mathrm{~Hz}$ y con un filtro de banda entre 10 a $500 \mathrm{~Hz}$, rectificada e integrada con una constante de tiempo de 20 milisegundos. La magnitud del parpadeo se define como la diferencia entre el mayor incremento de la actividad electro- miográfica del músculo orbicular ocurrida en el intervalo de tiempo entre 20 a 150 milisegundos inmediatamente después del estímulo de sobresalto, menos la media del EMG de los 25 milisegundos previos al inicio del sonido de sobresalto (Blumenthal et al., 2005).

Para corregir la variabilidad individual en la amplitud del reflejo de sobresalto, la magnitud de la respuesta se transformó a puntuaciones T en cada sujeto. A partir de lo anterior, el 1,8\% de todos los ensayos fueron excluidos debido a inestabilidad en la línea de base ( $>2$ DE por encima de la media de la línea de base de cada sujeto) o por una respuesta excesiva (outlier $>3$ DE por encima de la media de la magnitud del sobresalto para cada categoría de imagen en cada sujeto) (Cui et al., 2012).

La GSR se midió a través de dos electrodos de $8 \mathrm{~mm} \mathrm{Ag} / \mathrm{AgCl}$ colocados en la prominencia hipotenar de la mano no dominante. La tasa de muestreo utilizada fue de $100 \mathrm{~Hz}$ con un rango de respuesta de $0-40 \mu \mathrm{S}$. La respuesta electrodérmica se definió como la diferencia entre el punto más alto de la GSR entre los segundos 1 al 4 menos el promedio de la GSR durante el segundo previo al inicio de la imagen. Para normalizar la distribución de la GSR se utilizó la fórmula ( $\log [$ valor +1$])$ (Delgado et al., 2010).

\section{Consideraciones éticas}

El estudio fue aprobado por el comité de ética de la Universidad de San Buenaventura, Bogotá, y todos los participantes firmaron el consentimiento informado como requisito para participar en la investigación. A los participantes que hicieron parte del grupo de fumadores se les entregó después del estudio información sobre dónde buscar asesoría para la moderación o abandono del consumo de tabaco y a los no fumadores se les entregó información escrita sobre las consecuencias del consumo de tabaco. 


\section{Análisis estadístico}

Para evaluar el efecto del consumo de tabaco y el tipo de imagen sobre la magnitud del reflejo de sobresalto y la GSR, se aplicó dos ANOVA 2 $\times 4$ mixtas de medidas repetidas, como factor intersujeto "grupo" (fumador y no fumador), como factor intrasujeto "imagen" (apetitivas, neutrales, aversivas y tabaco), y como variables dependientes la magnitud del reflejo de sobresalto y la GSR. El factor de corrección Greenhouse-Geisser se utilizó para corregir cualquier violación de la esfericidad en el factor de medidas repetidas. Los análisis post hoc de los valores de las medias se llevaron a cabo a través de la comparación de pares con el factor Bonferroni. El nivel de significancia para todos los análisis fue 0,05 y el tamaño del efecto también se reporta $\left(\eta_{p}^{2}\right)$. Todos los análisis se realizaron en el software SPSS 20.0 para Windows.

\section{Resultados}

El ANOVA para la GSR no identificó ningún efecto principal ni de interacción significativos (todas las $p>0,37$ ). El ANOVA para el reflejo de sobresalto reveló un efecto principal significativo para imagen $\left(F_{(3,114)}=21,86, p<0,0001, \eta_{p}{ }^{2}=0,36\right)$. Como era de esperarse, las imágenes apetitivas inhibieron el reflejo de sobresalto en comparación con las demás categorías de imágenes (todas las $p<0,001)$ y las aversivas lo potenciaron en comparación con el resto de categorías (todas las $p<$ $0,02)$; no se encontraron diferencias significativas entre las imágenes neutrales y las de tabaco $(p=1)$.

Se encontró un efecto de interacción significativo entre grupo $\times$ imagen $\left(F_{(3,114)}=5,34, p=0,002\right.$, $\left.\eta_{\mathrm{p}}{ }^{2}=0,12\right)$. Las imágenes de tabaco inhibieron significativamente el reflejo de sobresalto en fumadores en comparación con los no fumadores $(p<0,0001)$ (ver figura 1). No se encontraron diferencias significativas entre grupos en ninguna otra categoría de imágenes (todas las $p>0,07$ ). Es también interesante que en el grupo de fuma- dores las imágenes de tabaco inhibieron el reflejo de sobresalto de la misma forma que las imágenes apetitivas $(p=1)$; por el contrario, en el grupo de no fumadores las imágenes apetitivas inhibieron el reflejo de sobresalto en comparación con las imágenes de tabaco $(p<0,0001)$.

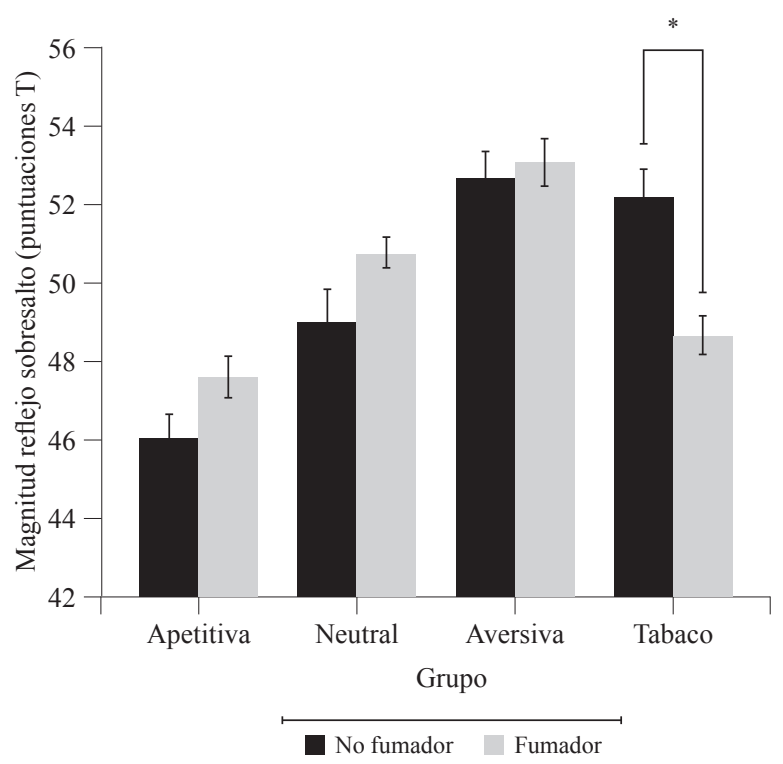

Figura 1. Reflejo de sobresalto en fumadores y no fumadores ante imágenes apetitivas, neutrales, aversivas y asociadas al tabaco. ${ }^{*} p<0,05$

\section{Discusión}

El objetivo de la presente investigación fue identificar la magnitud de la modulación del reflejo de sobresalto y la GSR ante imágenes asociadas al tabaco en fumadores y no fumadores. Los resultados muestran que los fumadores inhiben el reflejo de sobresalto ante los estímulos de tabaco en comparación con los no fumadores y, además, reaccionan ante los estímulos asociados al tabaco de la misma forma que ante estímulos apetitivos o placenteros, lo que demuestra en los fumadores la activación del sistema motivacional apetitivo ante los estímulos asociados al tabaco. Con respecto a la GSR, no se encuentran diferencias significativas entre fumadores y no fumadores. 
Estos resultados confirman lo encontrado en investigaciones anteriores (Cinciripini et al., 2006; Cui et al., 2012; Rehme et al., 2009), en las que se ha observado que los fumadores inhiben el reflejo de sobresalto ante estímulos visuales asociados al tabaco de la misma forma que lo hacen ante estímulos placenteros. Esto indica que los estímulos asociados al tabaco activan el sistema motivacional apetitivo en fumadores, lo que a su vez se asocia con conductas de aproximación (Bradley, 2009; Bradley et al., 2001; Bradley \& Lang, 2007). Estos datos aportan información explicativa a la conducta de fumar y a las recaídas que se dan durante el proceso de abandono (Gantiva \& Flórez-Alarcón, 2015; Shiffman et al., 2007), debido a que al activarse el sistema motivacional apetitivo ante estímulos o señales asociadas al tabaco, aumenta la probabilidad de aproximación al estímulo y, en este caso en particular, a la conducta de consumo.

Una nueva contribución de este estudio fue identificar que los fumadores inhiben el reflejo de sobresalto ante estímulos asociados al tabaco en comparación con los no fumadores. Este resultado es contradictorio con lo reportado por Rehme et al. (2009); sin embargo, en este estudio la metodología utilizada para evaluar la modulación del reflejo de sobresalto ante imágenes asociadas al tabaco fue a través de la comparación exclusivamente con imágenes neutrales, lo que podría explicar las diferencias en los resultados, ya que en nuestro estudio se comparó cada categoría de imagen por separado y entre ellas mismas en ambos grupos.

La activación del sistema motivacional apetitivo en fumadores ante estímulos asociados al tabaco, que se muestra en la inhibición del reflejo de sobresalto, es coherente con lo encontrado en investigaciones hechas con animales en las que, a través de la presentación de señales apetitivas, se reduce la respuesta de sobresalto. Este efecto es causado por el núcleo accumbens (Koch, Schmid, \& Schnitzler, 1996), estructura que influye directamente en el desarrollo de la adicción (Dani \& Harris, 2005), $y$ con estudios de neuroimagen en los que se ha observado que las señales de tabaco activan en los fumadores las zonas de recompensa ubicadas en el sistema mesolímbico (Vollstädt-Klein et al., 2011).

$\mathrm{La}$ ausencia de diferencias significativas en la GSR indica que los estímulos asociados al tabaco resultan apetitivos en los fumadores pero sin un aumento significativo en el arousal en comparación con los no fumadores. Estudios anteriores han reportado que los fumadores responden con mayor GSR ante videos relacionados al consumo de tabaco que ante videos con contenido neutral (Tong et al., 2007). Estos resultados contradictorios pueden explicarse por las diferentes modalidades de presentación de los estímulos visuales (imágenes versus videos), lo que sugiere que una secuencia de imágenes genera mayor arousal que una imagen estática.

Finalmente, es importante considerar algunas limitaciones del estudio. Primero, aunque se controló la inclusión de cada persona a los grupos por su historia e intensidad de consumo, es deseable para futuros estudios el uso de instrumentos de autoreporte que midan dependencia al tabaco. Segundo, es también recomendable que se controle el tiempo de abstinencia desde el último cigarrillo para el grupo de fumadores a través de la medición de $\mathrm{CO}$ en partículas por millón (ppm), pues existe evidencia de que el tiempo de abstinencia afecta la modulación del reflejo de sobresalto (Cinciripini et al., 2006).

\section{Referencias}

Blumenthal, T. D., Cuthbert, B. N., Filion, D. L., Hackley, S., Lipp, O. V., \& van Boxtel, A. (2005). Committee report: Guidelines for human startle eyeblink electromyographic studies. Psychophysiology, 42, 1-15.

Bradley, M. (2009). Natural selective attention: Orienting and emotion. Psychophysiology, 46, 1-11. doi: 10.1111/j.1469-8986.2008.00702.x Bradley, M., Codispoti, M., Cuthbert, B., \& Lang, P. J. (2001). Emotion and motivation I: Defensive 
and appetitive reactions in picture processing. Emotion, 1(3), 276-298. doi: 10.1037//15283542.1.3.276

Bradley, M. \& Lang, P. (2007). Emotion and motivation. En J. Cacioppo, L. Tassinary, \& G. Berntson (Eds.), The Handbook of Psychophysiology. Nueva York: Cambridge University Press.

Bradley, B., Mogg, K., Wright, T., \& Field, M. (2003). Attentional bias in drug dependence: vigilance for cigarette-related cues in smokers. Psychology of Addictive Behaviors, 17(1), 66-72. doi: 10.1037/0893-164X.17.1.66

Cinciripini, P. M., Robinson, J. D., Carter, B. L., Lam, C., Wu, X., de Moor, C. A., Wetter, D. et al. (2006). The effects of smoking deprivation and nicotine administration on emotional reactivity. Nicotine \& Tobacco Research, 8, 379-392.

Cui, Y., Robinson, J., Versace, F., Lam, C., Minnix, J., Karam-Hage, M., Cinciripini, P. et al. (2012). Differential cigarette-related startle cue reactivity among light, moderate, and heavy smokers. Addictive Behaviors, 37, 885-889. doi: 10.1016/j.addbeh.2012.02.003

Dani, J. A. \& Harris, R. A. (2005). Nicotine addiction and comorbidity with alcohol abuse and mental illness. Nature Neuroscience, 8, 1465-1470.

Delgado, L., Guerra, P., Perakakis, P., Vera, M., Reyes del Paso, G., \& Vila, J. (2010). Treating chronic worry: Psychological and physiological effects of a training programme based on mindfulness. Behaviour Research and Therapy, 48, 873-882. doi: 10.1016/j.brat.2010.05.012

Dempsey, J. P., Cohen, L. M., Hobson, V. L., \& Randall, P. K. (2007). Appetitive nature of drug cues re-confirmed with physiological measures and the potential role of stage of change. Psychopharmacology, 194, 253-260.

Gantiva, C., Díaz, D., Ospina, I., Gutiérrez, A., \& Romo-González, T. (2014). Modulation of the startle response in verbal aggressors: differences among stimuli with distinct affective social content. Psychology \& Neuroscience, 7(2), 221225. doi: 10.3922/j.psns.2014.016
Gantiva, C. \& Flórez-Alarcón, L. (2015). Entrevista motivacional en consumidores excesivos de alcohol: intervenciones efectivas para el aumento en la disposición al cambio. Psychologia: Avances de la Disciplina, 9(1), 83-91.

Gantiva, C., Guerra, P., \& Vila, J. (2011). Validación colombiana del sistema internacional de imágenes afectivas: evidencias del origen transcultural de la emoción. Acta Colombiana de Psicología, 14(2), 103-111.

Gantiva, C., Guerra, P., \& Vila, J. (2015). Modulación del reflejo de sobresalto en población colombiana: evidencia de la interacción entre emoción y motivación. Universitas Psychologica, 14(1), 157-164. doi: http://dx.doi.org/10.11144/Javeriana.upsy14-1.mrsp

Gantiva, C., Rodríguez, M., Arias, M., \& Rubio, E. (2012a). Dimensiones de la emoción durante el proceso de abandono del consumo de tabaco: un apoyo a la visión motivacional del cambio. Diversitas: Perspectivas en Psicología, 8(2), 309-317.

Gantiva, C., Rodríguez, M., Arias, M., Rubio, E., Guerra, P., \& Vila, J. (2012b). Diseño y validación de un sistema de imágenes afectivas relacionadas con el consumo de tabaco en población colombiana. Pensamiento Psicológico, 10(2), 113-122.

Koch, M., Schmid, A., \& Schnitzler, H. U. (1996). Pleasure-attenuation of startle is disrupted by lesions of the nucleus accumbens. Neuroreport, 7, 1442-1 446.

Lang, P. J. (1995). The emotion probe: Studies of motivation and attention. American Psychologist, 50, 372-385.

Lang, P. J. (2010). Emotion and motivation: Toward consensus definitions and a common research purpose. Emotion Review, 2(3), 229-233. doi: 10.1177/1754073910361984

Lang, P., Bradley, M., \& Cuthbert, B. (2008). International affective picture system (IAPS): Affective ratings of pictures and instruction 
manual. Technical Report A-8. Gainesville, FL: University of Florida.

Loeber, S., Croissant, B., Nakovics, H., Zimmer, A., Georgi, A., Klein, S., Flor, H. et al. (2007). The startle reflex in alcohol-dependent patients: Changes after cognitive-behavioral therapy and predictive validity for drinking behavior. Psychotherapy and Psychosomatics, 76(6), 385390. doi: 10.1159/000107567

Marín Mayor, M., Jurado-Barba, R., Martínez-Grass, I., Ponce Alfaro, G., \& Rubio Valladolid, G. (2014). La respuesta de sobresalto y la inhibición prepulso en los trastornos por uso de alcohol: implicaciones para la práctica clínica. Clínica y Salud, 25(3), 147-155. doi: http://dx. doi.org/10.1016/j.clysa.2014.10.003

Muñoz, M., Viedma-del-Jesus, M., Fernández-Santaella, M., Peralta-Ramírez, M., Cepeda-Benito, A., \& Vila, J. (2010). Assessment of tobacco craving by means of the affective image visualization paradigm. Motivation and Emotion, 34, 93-103. doi: 10.1007/s11031-009-9145-1

Organización Mundial de la Salud (OMS). (2011). Informe OMS sobre la epidemia mundial de tabaquismo, 2011. Recuperado de http://whqlibdoc. who.int/hq/2011/WHO_NMH_TFI_11.3_spa. pdf?ua $=1$

Organización Mundial de la Salud (OMS). (2014). Prevalence of tobacco use among adults and adolescents. Recuperado de http://gamapserver.who.int/gho/interactive_charts/tobacco/use/ atlas.html
Rehme, A. K., Frommann, I., Peters, S., Block, V., Bludau, J., Quednow, B. B., Wagner, M. et al. (2009). Startle cue-reactivity differentiates between light and heavy smokers. Addiction, 104(10), 1757-1 764.

Shiffman, S., Balabanis, M. H., Gwaltney, C. J., Paty, J. A., Gnys, M., Kassel, J. D., Paton, S. M. et al. (2007). Prediction of lapse from associations between smoking and situational antecedents assessed by ecological momentary assessment. Drug and Alcohol Dependence, 91, 159-168.

Tong, C., Bovbjerg, D., \& Erblich, J. (2007). Smoking-related videos for use in cue-induced craving paradigms. Addictive Behaviors, 32, 3 034-3 044.

Versace, F., Minnix, J. A., Robinson, J. D., Lam, C. Y., Brown, V. L., \& Cinciripini, P. M. (2011). Brain reactivity to emotional, neutral and cigarette-related stimuli in smokers. Addiction Biology, 16, 296-307.

Versace, F., Robinson, J. D., Lam, C. Y., Minnix, J. A., Brown, V. L., Carter, B. L., Cinciripini, P. M. et al. (2010). Cigarette cues capture smokers' attention: Evidence from event-related potentials. Psychophysiology, 47, 435-441.

Vollstädt-Klein, S., Kobiella, A., Bühler, M., Graf, C., Fehr, C., Mann, K., \& Smolka M. N. (2011). Severity of dependence modulates smokers' neuronal cue reactivity and cigarette craving elicited by tobacco advertisement. Addiction Biology, 16, 166-175.
Fecha de recepción: abril 13, 2015 Fecha de aceptación: octubre 18, 2015 